Document downloaded from:

http://hdl.handle.net/10251/145985

This paper must be cited as:

Oser, D.; Pérez-Galacho, D.; Alonso-Ramos, C.; Le Roux, X.; Tanzilli, S.; Vivien, L.; Labonte, L.... (15-0). Subwavelength engineering and asymmetry: two efficient tools for subnanometer-bandwidth silicon Bragg filters. Optics Letters. 43(14):3208-3211. https://doi.org/10.1364/OL.43.003208

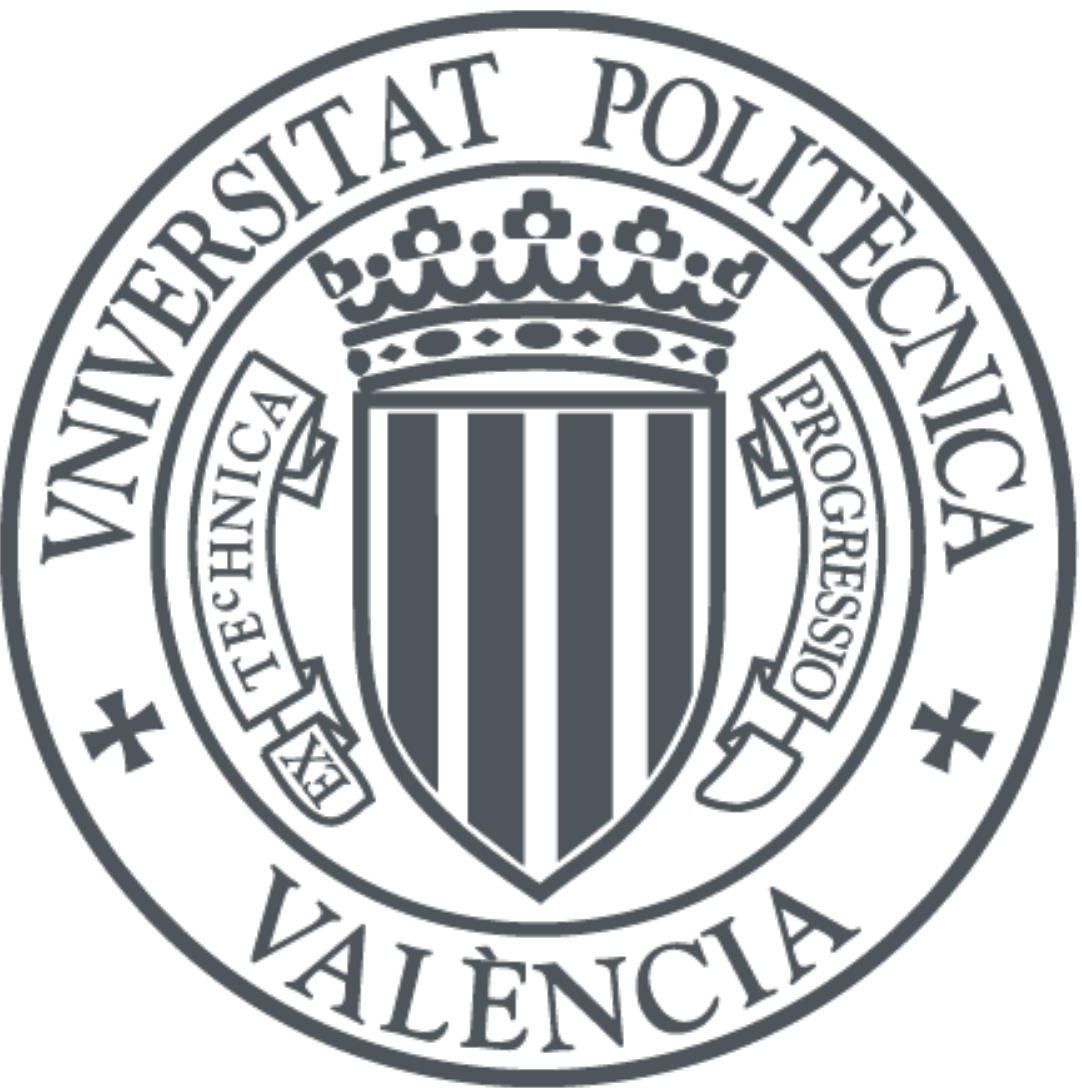

The final publication is available at

https://doi.org/10.1364/OL.43.003208

Copyright The Optical Society

Additional Information 


\title{
Subwavelength engineering and asymmetry: two efficient tools for sub-nanometer-bandwidth silicon Bragg filters
}

\author{
Dorian Oser ${ }^{1,}{ }^{*}$, Diego Perez-Galacho ${ }^{1,4}$, Carlos Alonso-Ramos ${ }^{1}$, Xavier Le $^{\circ}$

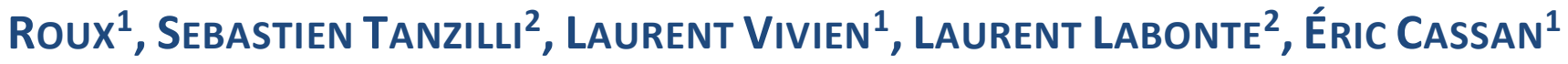 \\ ${ }^{1}$ Centre de Nanosciences et de Nanotechnologies, Université Paris-Saclay, Orsay site, F91405 Orsay cedex, France \\ 2Université côte d'Azur, CNRS, Institut de Physique de Nice, Parc Valrose, 06108 Nice cedex 2, France \\ *Corresponding author: dorian.oser@c2n.upsaclay.fr
}

Received XX Month XXXX; revised XX Month, XXXX; accepted XX Month XXXX; posted XX Month XXXX (Doc. ID XXXXX); published XX Month XXXX

\begin{abstract}
Bragg filters stand as a key building blocks of the siliconon-insulator (SOI) photonics platform, allowing the implementation of advanced on-chip signal manipulation. However, achieving narrowband Bragg filters with large rejection levels is often hindered by fabrication constraints and imperfections. Here, we show that the combination of single-side corrugation asymmetry and subwavelength engineering provides narrowband response with large corrugations, overcoming minimum feature size constraints of conventional Si Bragg filters. We comprehensively study the impact of the corrugation asymmetry in conventional and subwavelength singleetched SOI Bragg filters, showing their potential for bandwidth reduction. Finally, we experimentally demonstrate a novel subwavelength geometry, based on shifted corrugation teeth, achieving null-to-null bandwidths and rejections of $0.8 \mathrm{~nm}$ and $40 \mathrm{~dB}$ for the symmetric configuration, and $0.6 \mathrm{~nm}$ and $15 \mathrm{~dB}$ for the asymmetric case.
\end{abstract}

OCIS codes: (230.1480) Bragg reflectors; (050.6624) Subwavelength structures; (160.3130) Integrated optics materials.

http://dx.doi.org/10.1364/OL.99.099999

The Silicon-on-insulator (SOI) platform provides miniaturized optical circuits that can be fabricated in already existing CMOS facilities [1]. These high-performance photonic circuits have an exciting potential for a plethora of applications [2,3]. Notably, Bragg filters are fundamental components of the SOI library, allowing wavelength selection [5], on-chip rejection of pump lasers [5], sensing [3], mode selection in laser cavities [6], as well as on-chip single photon manipulation [4]. Here, we show that for a given minimum feature size, single-side (asymmetric) corrugation, allows straightforward reduction of filter bandwidth for both conventional and subwavelength-engineered filters. We also show that the degrees of freedom released by the combination of corrugation asymmetry and subwavelength engineering, open a new route for the implementation of highly tailorable filter responses. Based on this concept, we experimentally demonstrate Bragg filters with different bandwidth-rejection features, ranging from ultra-narrow (lower than one nanometer) to ultra-deep rejection operation (around $50 \mathrm{~dB}$ ).

A waveguide Bragg filter is a periodic structure that reflects incoming light at, and around, a specific wavelength. The three main performance parameters of Bragg filters are the central wavelength $\left(\lambda_{0}\right)$, the reflection level $(R)$ and the null-to-null bandwidth $(\Delta \lambda)$. The central wavelength is determined by the pitch $(\Lambda)$ and the effective index of the mode $n_{\text {eff }}$, as $\lambda_{0}=2 \Lambda n_{\text {eff }}$ [6]. On the other hand, the bandwidth and rejection level are determined by

$$
\begin{gathered}
\Delta \lambda=\frac{\lambda_{0}^{2}}{\pi n_{g}} \sqrt{\kappa^{2}+\frac{\pi^{2}}{L^{2}},} \\
R=\tanh ^{2}(\kappa L),
\end{gathered}
$$

where $\kappa$ is the coupling coefficient (coupling rate between forward and backward propagating modes of the periodic structure), $L$ the filter length, and $n_{g}$ the group index. These equations, derived from the coupled mode theory [7], state that both filter bandwidth and rejection level mostly depend on the index modulation (through $\kappa$ ) and the grating total length $L$ [7]. The coupling coefficient is proportional to the index modulation [8]. Therefore, a narrowband filter with a few nanometer bandwidth requires weak index modulations. Conversely, wideband filters require strong modulations. The minimum achievable bandwidth is ultimately limited by the weakest index modulation that can be effectively implemented. In most Bragg filter configurations, modulation is related to the corrugation width. Hence, due to the high refractive index difference between silicon and $\mathrm{SiO}_{2}$, conventional SOI Bragg filters require $\sim 10 \mathrm{~nm}$ corrugations to yield narrow-band 
operation [8], complicating the device fabrication. Bragg filters based on shallow-etched rib geometries, achieve narrow bandwidths with relaxed corrugation widths exceeding $80 \mathrm{~nm}$ [9]. However, they rely on a two-step fabrication process that compromises the simplicity and the yield of this approach. Modulation of the waveguide cladding has shown moderate bandwidths below $10 \mathrm{~nm}$ [10]. Contra-directional cross-mode coupling in asymmetrically corrugated multi-mode $\mathrm{Si}$ waveguides yielded narrow-band rejection with corrugation widths larger than $100 \mathrm{~nm}$ [11]. But, this option required a multimode waveguide configuration. It was also demonstrated that reducing mode confinement by changing polarization from transverse-electric (TE) to transverse-magnetic (TM), substantial reduces filter bandwidth [12]. The corrugation width is limited by minimum feature size of the lithographic process, which is usually around $50 \mathrm{~nm}$ in electron beam and $100 \mathrm{~nm}$ in optical lithography, respectively. This limits the minimum bandwidth of conventional Bragg filters.

Another approach to reduce the filter bandwidth, keeping relaxed minimum feature size constraints, is subwavelength engineering of the Bragg unit cell $[11,13,14]$. Subwavelength waveguides exploit periodic patterns with a periodicity smaller than half of the wavelength to tune index or dispersion properties $[15,16]$. In subwavelength engineered Bragg filters, the total period is divided in two subwavelength sub-periods that mimic the narrow and wide parts of the conventional Bragg geometry. The pattern variation needed to induce this spatially-averaged index modulation (e.g. the difference between two teeth widths) is limited by the lithography precision. As it is usually 10 times smaller than its minimum feature size. Hence, this configuration allows weak index modulations with comparatively large corrugations, relaxing minimum feature size constraints.

In this work, we compared conventional Bragg filters with state-ofthe-art subwavelength double-width corrugation Bragg filters (DWCBF) [9]. We also report on a novel corrugation-shifted Bragg filter (CSBF) geometry. Conventional Bragg filters rely on a periodic corrugation of the waveguide width $W$ (see Fig. 1(a)). As depicted in Fig. 1(a) incident light propagates along the $\mathrm{z}$ axis. The TE polarization is aligned with the xaxis and transverse-magnetic (TM) polarization is perpendicular to the $\mathrm{xz}$ plane. In the DWCBF configuration, the index modulation is realized by the relative width difference between the two subwavelength teeth $d W \neq 0$ (Fig. 2(a)). Conversely, in the CSBF approach, both subwavelength teeth have the same width, with the position of one of them longitudinally shifted. This can be seen as a compression of the sub-period $d L=$ $\Lambda_{1}-\Lambda_{2} \neq 0$ (with $\Lambda=\Lambda_{1}+\Lambda_{2}$ ) (Fig. 2(a)). This way, the index modulation is implemented maintaining the same amount of $\mathrm{Si}$ in both sub-periods. As both teeth may be affected by proximity effects in the same fashion, this geometry could provide relax fabrication tolerances. Nevertheless, verifying this improved robustness would require statistical experimental analysis, which is out of the scope of this work. Wealso studied the single-side asymmetric configuration for these three kinds of Bragg filters, conventional (Fig. 1(b)), double-width (Fig. 2(c)), and shifted (Fig. 2(d)).

The filters presented here are designed considering TE polarization, a 220-nm-thick Si layer and PMMA cladding. Minimum feature size of $50 \mathrm{~nm}$ is set for compatibility with our electron beam lithography. As a first approximation to the problem, we used coupled mode theory to estimate the filter bandwidth from the coupling coefficient and group index. First, we studied the conventional geometry depicted in Fig. 1(a). We computed the effective index difference
$\Delta n_{\text {eff }}$ between the modes of the wide and narrow regions and estimated the coupling coefficient as $\kappa=2 \lambda_{0} / \Delta n_{\text {eff }}$. We considered a mode group index $n_{g}$ of 4.2. Then, we calculated a bandwidth of $23 \mathrm{~nm}$ for a given central width, $W=400 \mathrm{~nm}$, and a corrugation width of $50 \mathrm{~nm}$. One way to reduce the filter bandwidth keeping the same minimum feature size is to implement the singleside asymmetric corrugation. This geometry, presented in Fig. 1(b), halves the $\Delta n_{e f f}$, providing a two-fold reduction in filter bandwidth. For the DCWBF, we estimated $\Delta n_{\text {eff }}$ by zero-order approximation of the subwavelength index [17]. For the CSBF, we used 2.5D FDTD simulation (Lumerical Mode Solution) to estimate the bandwidth. In both cases, we calculated a bandwidth of $\sim 6 \mathrm{~nm}$ for differential waveguide width and longitudinal shift ( $d W$ and $d L$ ) of $5 \mathrm{~nm}$ with a group index of $n_{g}=3.5$. This represents a bandwidth reduction by almost a factor of 4 , compared to the conventional approach.
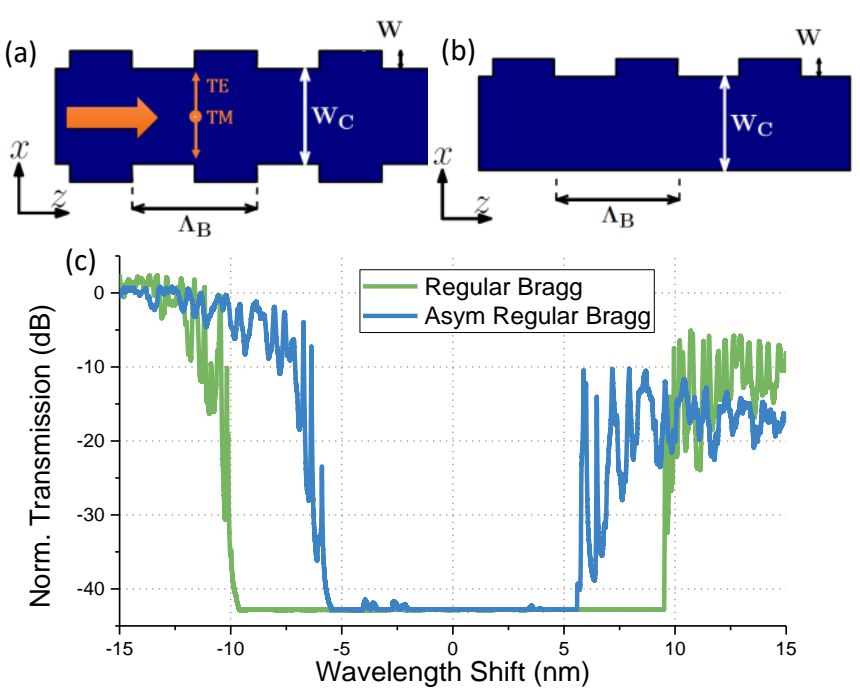

Fig. 1: Top-view schematics of conventional Bragg geometries, (a) regular Bragg filter, (b) asymmetric regular Bragg filter. (c) Measured TE transmission spectra for the two geometries with a pitch of $340 \mathrm{~nm}$, an average waveguide width of $400 \mathrm{~nm}$, and a corrugation of $50 \mathrm{~nm}$. The minimum of $-43 \mathrm{~dB}$ corresponds to the saturation of the photo-detector. The central wavelength of the regular Bragg is $1542 \mathrm{~nm}$ and the central wavelength of the asymmetric regular Bragg is $1550 \mathrm{~nm}$.

The realization of integrated Bragg filters simultaneously presenting high selectivity and strong rejection is limited in practice by the technological limitations. Hence, we decided to experimentally compare the performance of the different filter geometries proposed here.

We fabricated a series of Bragg filters following the different strategies illustrated in Figs. 1 and 2. We used SOI wafers comprising a $220 \mathrm{~nm}$ thick silicon and a $2 \mu \mathrm{m}$ buried oxide layer. Electron beam lithography (Nanobeam NB-4 system $80 \mathrm{kV}$, with a step size of $5 \mathrm{~nm}$ ) and dry and inductively coupled plasma etching (SF6 gas) were used to define the patterns. A cladding of PMMA was added at the end of the process to protect the devices. Subwavelength fiber-chip grating couplers were used to inject and extract TE-polarized light with standard single mode (SMF-28) optical fibers [18]. These grating couplers were optimized to reduce Fabry-Perot ripples in the collected signal for an easier analysis of the transmission spectrum. We measured the transmission 
spectrum of the filters using a tunable laser and an associated data acquisition system (Yenista tunics and CT400). We used a polarization rotator to the inject TE-polarized light into the gratings. All filter spectra are normalized to the maximum transmission to remove insertion loss from the fiber-chip gratings.

The scanning electron images in Fig. 3 show that the fabricated structures exhibit rounded corners, which mainly results in a blueshift of the resonant wavelength and a slight reduction of the coupling coefficient. For comparison, all filters have an average width of $450 \mathrm{~nm}$ (Fig. 3). The periods ( $\Lambda$ ) yielding Bragg resonance around $1550 \mathrm{~nm}$ wavelength are $340 \mathrm{~nm}$ and $320 \mathrm{~nm}$ for the subwavelength and regular gratings, respectively. For the regular Bragg filters, we fixed a duty cycle of $50 \%$ and a waveguide width of $W c=400 \mathrm{~nm}$. We then varied the corrugation between $50 \mathrm{~nm}$ (which is our minimum feature size) and $70 \mathrm{~nm}$. For the DWCBF, we fixed a duty cycle of $50 \%$ in the sub-periods, a waveguide width of $W c=300 \mathrm{~nm}$ and a corrugation of $W=150 \mathrm{~nm}$. We implemented differential waveguide widths of $\mathrm{d} W=5 \mathrm{~nm}, 10 \mathrm{~nm}$, and $15 \mathrm{~nm}$. For the CSBF, we used a waveguide width of $W c=300 \mathrm{~nm}$, but we set the corrugation to $W=150 \mathrm{~nm}$. We then scanned the longitudinal shift $d L$ between $5 \mathrm{~nm}$ and $15 \mathrm{~nm}$.
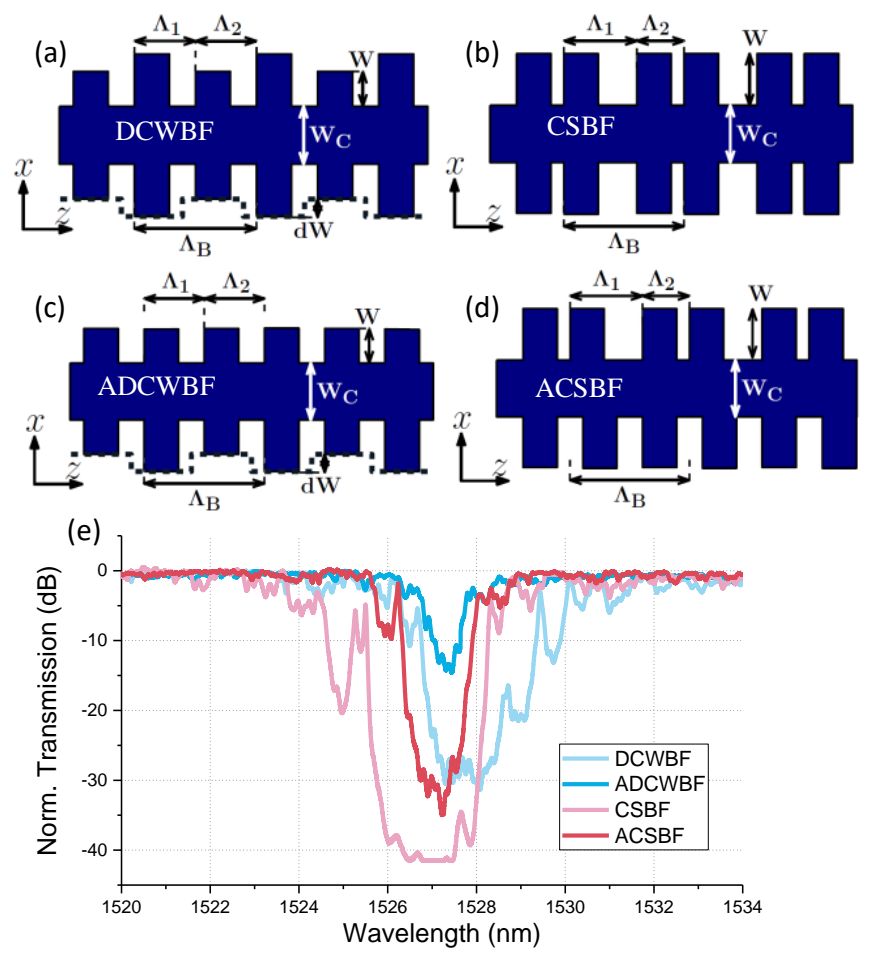

Fig. 2: Top-view schematics of the subwavelength Bragg geometries, (a) double corrugation width Bragg filter (DCWBF), (b) corrugationshifted Bragg filter (CSBF), (c) asymmetric double corrugation width Bragg filter (ADCWBF), and (d) asymmetric corrugation-shifted Bragg filter (ACSBF). (e) Measured transmission spectra of the 4 geometries with a pitch of $320 \mathrm{~nm}, W c=300 \mathrm{~nm}, \mathrm{~W}=150 \mathrm{~nm}$, length of $500 \mu \mathrm{m}$, and $d L=d W=10 \mathrm{~nm}$.

As shown in Fig. 1(c), the conventional filters with a corrugation of $50 \mathrm{~nm}$ have wide measured bandwidth of $\sim 20 \mathrm{~nm}$. The asymmetric single-side corrugation allowed an almost two-fold bandwidth reduction, yielding $\sim 12 \mathrm{~nm}$.

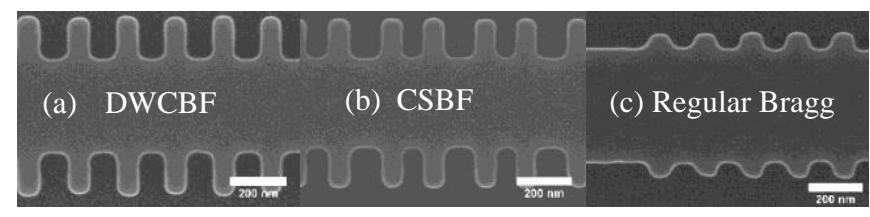

Fig. 3: Scanning electron microscope images of three Bragg filters fabricated with electron beam lithography. (a) is the longitudinally shifted Bragg filter, (b) is the two width Bragg filter, and (c) is a conventional Bragg filter of $50 \mathrm{~nm}$ corrugation.

Figure 2(e) compares the measured bandwidths of subwavelength engineered filters in symmetric and asymmetric configurations, with differential corrugation ( $d W$ and $d L$ ) of $10 \mathrm{~nm}$. Both symmetric designs provide a bandwidth reduction more than 5 times greater compared to the conventional design. Again, the asymmetric design yields a substantial bandwidth reduction (from $4.2 \mathrm{~nm}$ to $1.8 \mathrm{~nm}$ with the ACSBF).
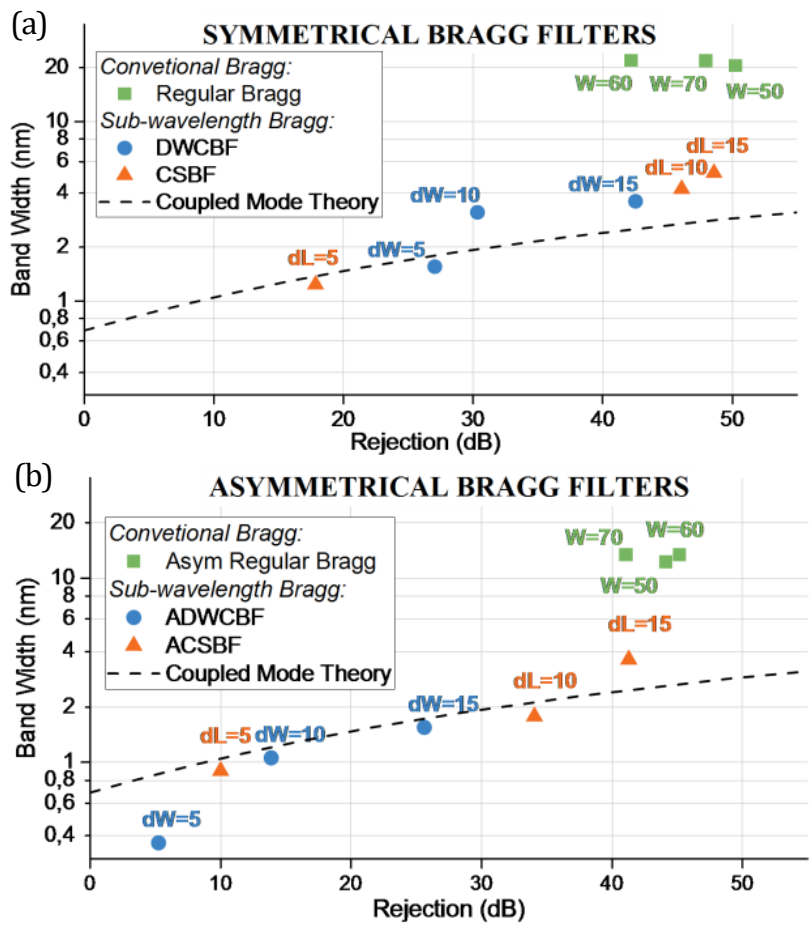

Fig. 4: Experimental bandwidth and rejection of different Bragg filters based on (a) symmetric, and (b) asymmetric geometries. All the studied Bragg filters have a length of $500 \mu \mathrm{m}$. Labels indicate the different geometries, following notations in Fig. 1 and 2.

As a general summary, Fig. 4 shows the performance of all the filter geometries studied here in terms of bandwidth and rejection levels. The length of all filters is $L=500 \mu \mathrm{m}$, ensuring reasonably compact devices and measurable optical resonances. As a guide for analysis, we also plot the theoretical bandwidth-rejection curve (dashed line in Fig. 4(a) and 4(b)), estimated from eqs. (1) and (2) by varying the coupling coefficient, $\kappa$, with a group index of $n_{g}=3.5$, which models subwavelength filters [8].

In all cases, the asymmetric filter configuration provides a great bandwidth reduction, widening the design space for a given minimum feature size. For instance, both the symmetric DWCBF with $d W=5 \mathrm{~nm}$ and the asymmetric ADWCBF with $d W=15 \mathrm{~nm}$, experimentally provide an order of magnitude bandwidth 
reduction, compared with the regular filter geometries. However, the asymmetric configuration relies in a three-times wider differential corrugation, relaxing lithography resolution requirements. This way, the combination of subwavelength engineering and corrugation asymmetry provides a useful design flexibility. It allows for ultra-narrowband response with $400 \mathrm{pm}$ width, or remarkably large rejection levels, close to $50 \mathrm{~dB}$. Yet it provides a five-times bandwidth reduction, compared to conventional counterparts. The behavior of subwavelength filters follows the trends predicted by coupled mode theory, with experimental points following the theoretical curves (dashed lines in Fig. 4). However, conventional designs presented here are considerably deviated. The reason for this is that, while theory predicts a rejection exceeding $100 \mathrm{~dB}$ or even $200 \mathrm{~dB}$, measured rejection is limited to $\sim 50 \mathrm{~dB}$, mainly due to phase errors arising from small fabrication imperfections.

To demonstrate the potential of the proposed shifted subwavelength filter, we fabricated symmetric (CSBF) and asymmetric configurations (ACSBF) with $d L=5 \mathrm{~nm}$ and $1 \mathrm{~mm}$ length. Figure 5 shows the transmission and reflection spectra for the two filters. Back-reflections are collected using a circulator connected to the input fiber. The measured reflection spectra are processed with the minimum phase technique [19] to remove the direct reflection from the input grating couplers. Both filters yield sub-nanometer bandwidth. The symmetric geometry exhibits a null-to-null bandwidth of $0.8 \mathrm{~nm}$ with a rejection exceeding $40 \mathrm{~dB}$, while the asymmetric design provides a bandwidth of $0.6 \mathrm{~nm}$ and a rejection of $\sim 15 \mathrm{~dB}$.
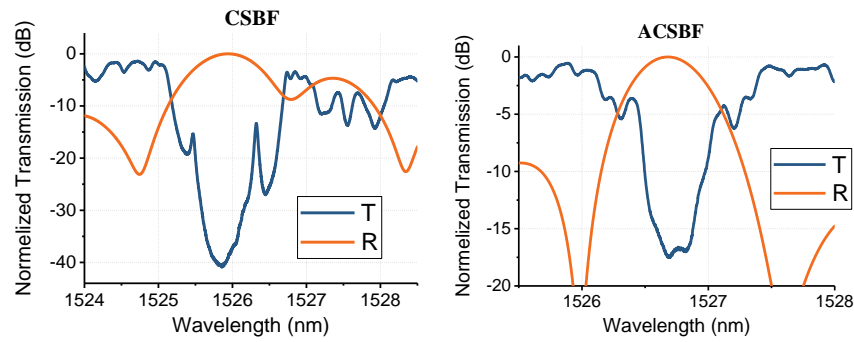

Fig. 5: Transmission (T) and reflection (R) spectra of the corrugated shifted Bragg filter (CSBF) and the asymmetric corrugated shifted Bragg filter (ACSBF), both with a shift $d L$ of $5 \mathrm{~nm}$ and a length of $1 \mathrm{~mm}$.

In conclusion, we have experimentally demonstrated that the combination of corrugation asymmetry and subwavelength engineering provides a great flexibility for the implementation of high-performance SOI Bragg filters. Thanks to proper geometry design, we showed measured filter responses ranging from ultranarrowband, with a few-hundred pm bandwidth, to ultra-deep operation, with rejection levels of $\sim 50 \mathrm{~dB}$. We show that, for a minimum feature of $50 \mathrm{~nm}$, the subwavelength filters presented here yield one order of magnitude bandwidth reduction compared with conventional counterparts. We also demonstrated filters with an excellent trade-off between bandwidth and rejection with subnanometer null-to-null bandwidth and depth exceeding $40 \mathrm{~dB}$. This is, to the best of our knowledge, the largest rejection experimentally demonstrated for TE-polarized sub-wavelength Bragg filters with sub-nanometer bandwidth. All these subwavelength filters were implemented in a single-etch step process with minimum feature size of $70 \mathrm{~nm}$, well within the capabilities of standard electron-beam lithography. These results demonstrate the potential of the proposed approach and open a new route for the implementation and exploitation of high-performance, tailorable Bragg filters on the SOI platform.

Funding. Agence National pour la Recherche, Project SITQOM (DS0710).

Acknowledgment. The sample fabrication has been performed at the Plateforme de Micro-Nano-Technologie/C2N, which is partially funded by the "Conseil Général de l'Essonne". This work as partly supported by the French RENATECH network.

$\triangle$ Diego Pérez-Galacho is currently at Photonics Reseach Labs, iTEAM Research Institute, Universitat Politecnica de Valencia, Spain.

\section{References}

1. F. Boeuf, S. Crémer, E. Temporiti, M. Ferè, M. Shaw, C. Baudot, N. Vulliet, T. Pinguet, A. Mekis, G. Masini, H. Petiton, P. L. Maitre, M. Traldi, and L. Maggi, J. Light. Technol. 34, 286 (2016).

2. A. Fernández Gavela, D. Grajales García, J. C. Ramirez, and L. M. Lechuga, Sensors 16, 285 (2016).

3. S. Janz, D.-X. Xu, M. Vachon, N. Sabourin, P. Cheben, H. Mclntosh, H. Ding, S. Wang, J. H. Schmid, A. Delâge, J. Lapointe, A. Densmore, R. Ma, W. Sinclair, S. M. Logan, R. MacKenzie, Q. Y. Liu, D. Zhang, G. Lopinski, O. Mozenson, M. Gilmour, and H. Tabor, Opt. Express 21, 4623 (2013).

4. S. Azzini, D. Grassani, M. J. Strain, M. Sorel, L. G. Helt, J. E. Sipe, M. Liscidini, M. Galli, and D. Bajoni, Opt. Express 20, 23100 (2012).

5. L. Pavesi and D. J. Lockwood, eds., Silicon Photonics III, Topics in Applied Physics (Springer Berlin Heidelberg, 2016), Vol. 122.

6. C. A. Zaugg, Z. Sun, V. J. Wittwer, D. Popa, S. Milana, T. S. Kulmala, R. S. Sundaram, M. Mangold, O. D. Sieber, M. Golling, Y. Lee, J. H. Ahn, A. C. Ferrari, and U. Keller, Opt. Express 21, 31548 (2013).

7. D. C. Flanders, H. Kogelnik, R. V. Schmidt, and C. V. Shank, Appl. Phys. Lett. 24, 194 (1974).

8. D. Pérez-Galacho, C. Alonso-Ramos, F. Mazeas, X. L. Roux, D. Oser, W. Zhang, D. Marris-Morini, L. Labonté, S. Tanzilli, É. Cassan, and L. Vivien, Opt. Lett. 42, 1468 (2017).

9. X. Wang, W. Shi, R. Vafaei, N. A. F. Jaeger, and L. Chrostowski IEEE Photonics Technol. Lett. 23, 290 (2011).

10. D. T. H. Tan, K. Ikeda, and Y. Fainman, Opt. Lett. 34, 1357 (2009).

11. H. Qiu, J. Jiang, P. Yu, T. Dai, J. Yang, H. Yu, and X. Jiang, Opt. Lett. 41, 2450 (2016).

12. Z. Chen, J. Flueckiger, X. Wang, F. Zhang, H. Yun, Z. Lu, M. Caverley, Y. Wang, N. A. F. Jaeger, and L. Chrostowski, Opt. Express 23, 25295 (2015).

13. J. Wang, I. Glesk, and L. R. Chen, Electron. Lett. 51, 712 (2015).

14. J. Čtyroký, J. G. Wangüemert-Pérez, P. Kwiecien, I. Richter, J. Litvik, J. H. Schmid, Í. Molina-Fernández, A. Ortega-Moñux, M. Dado, and P. Cheben, Opt. Express 26, 179 (2018).

15. W. C. Jiang, X. Lu, J. Zhang, O. Painter, and Q. Lin Opt. Express 23, 20884 (2015).

16. R. Halir, P. J. Bock, P. Cheben, A. Ortega-Moñux, C. Alonso-Ramos, J. H. Schmid, J. Lapointe, D.-X. Xu, J. G. Wangüemert-Pérez, Í. MolinaFernández, and S. Janz, Laser Photonics Rev. 9, 25 (2015).

17. S.M. Rytov, Sov. Phys. 2, 466 (1956)

18. D. Benedikovic, P. Cheben, J. H. Schmid, D.-X. Xu, B. Lamontagne,S. Wang, J. Lapointe, R. Halir, A. Ortega-Moñux, S. Janz, and M. Dado, Opt. Express 23, 22628 (2015).

19. R. Halir, I. Molina-Fernández, J. G. Wangüemert-Pérez, A. Ortega-Moñux, J. de-Oliva-Rubio, and P. Chenben, Opt. Express, 17, 8349 (2009). 